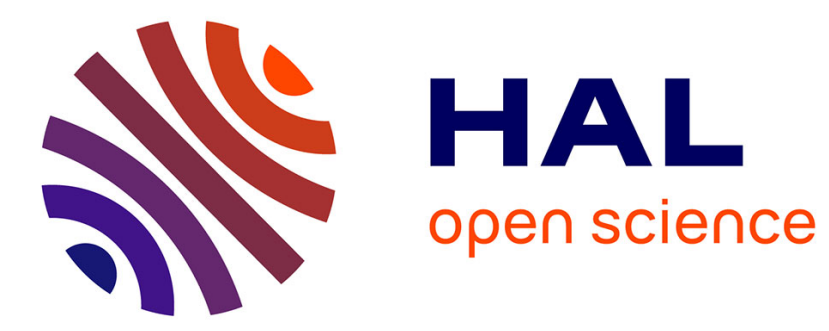

\title{
Mesure directe de l'électromigration sur des couches métalliques à l'aide de traceurs radioactifs
}

\author{
K.V. Reddy, J.J.B. Prasad, F. Bénière
}

\section{To cite this version:}

K.V. Reddy, J.J.B. Prasad, F. Bénière. Mesure directe de l'électromigration sur des couches métalliques à l'aide de traceurs radioactifs. Revue de Physique Appliquée, 1983, 18 (10), pp.613-617. 10.1051/rphysap:019830018010061300 . jpa-00245122

\section{HAL Id: jpa-00245122 https://hal.science/jpa-00245122}

Submitted on 1 Jan 1983

HAL is a multi-disciplinary open access archive for the deposit and dissemination of scientific research documents, whether they are published or not. The documents may come from teaching and research institutions in France or abroad, or from public or private research centers.
L'archive ouverte pluridisciplinaire HAL, est destinée au dépôt et à la diffusion de documents scientifiques de niveau recherche, publiés ou non, émanant des établissements d'enseignement et de recherche français ou étrangers, des laboratoires publics ou privés. 


\title{
Mesure directe de l'électromigration sur des couches métalliques à l'aide de traceurs radioactifs
}

\author{
K. V. Reddy, J. J. B. Prasad \\ Department of Physics, Indian Institute of Technology, Madras 600036, Inde \\ et $\mathrm{F}$. Bénière \\ Science des Matériaux, Université de Rennes I, 35042 Rennes, France
}

(Reçu le 22 mars 1983, révisé le 20 mai, accepté le 17 juin 1983)

\begin{abstract}
Résumé. - Nous avons mis au point une méthode très précise de mesure du déplacement par électromigration des atomes dans des rubans métalliques. Elle est basée sur le marquage isotopique, l'autoradiographie et le comptage de la radioactivité à travers une fente. L'incertitude relative sur le seul déplacement est égale à $1 \%$. La méthode a été testée avec succès sur l'électromigration dans l'indium en couche mince. La mobilité a pu ainsi être mesurée entre 100 et $150^{\circ} \mathrm{C}$ où elle suit la loi d'Arrhénius : $\mu T=3,69 \times 10^{21} \exp (-2,00 / k T) \mathrm{V}^{-1} \cdot \mathrm{cm}^{2} \cdot \mathrm{s}^{-1} \cdot \mathrm{K}$.

Abstract. - We have designed a very accurate method of measurement of the electromigration shift of atoms in metallic films. It is based on the isotopic labelling, autoradiography and the selective counting of radioactivity through a slit. The relative error on the shift only is equal to $1 \%$. The method has been successfully tested on electromigration in indium thin films. The mobility has been measured between 100 and $150{ }^{\circ} \mathrm{C}$ where it follows the Arrhenius law : $\mu T=3.69 \times 10^{21} \exp (-2.00 / k T) \mathrm{V}^{-1} \cdot \mathrm{cm}^{2} \cdot \mathrm{s}^{-1} . \mathrm{K}$.
\end{abstract}

\section{Introduction.}

L'électromigration sur des couches métalliques minces a pris une importance toute particulière en microélectronique où les contacts électriques sont réalisés par métallisation. Les couches ont une épaisseur inférieure à $1 \mu$. Conséquemment, les densités de courant sont supérieures à $10^{4} \mathrm{~A} . \mathrm{cm}^{-2}$ [1] et peuvent entraîner un déplacement macroscopique de matière et même la coupure du contact. Cet effet a été très étudié pour l'aluminium [2,3], l'or [4, 5] et l'étain [6, 7]. Les moyens de caractérisation les plus employés sont le microscope électronique à balayage et la microsonde électronique $[3,8]$, particulièrement dans le cas des alliages. Il faut en effet noter que depuis la découverte de D'Heurle [9] de la réduction du phénomène d'électromigration par incorporation d'une faible teneur d'atomes étrangers, les contacts sont le plus souvent des alliages, par exemple $\mathrm{Al}(99 \%)-\mathrm{Cu}(1 \%)$. La microsonde électronique est remarquablement bien adaptée à l'étude du déplacement des atomes de soluté dans le solvant [10] mais devient inopérante dans le cas d'un métal pur où il faut étudier le transport des atomes de soluté. On peut alors faire appel au marquage par des isotopes radioactifs dont le transport est suivi par les techniques radioactives $[6,11]$. Citons parmi celles-ci le découpage mécanique avec comptage de la radioactivité de chaque section $[10,12]$ ainsi que le balayage d'un compteur de radioactivité avec un diaphragme adéquat $[13,14]$. Cependant, ces travaux se rapportent à la mesure du déplacement par électromigration d'atomes de soluté dans des couches métalliques minces. Au contraire, le présent travail a trait à la mesure de l'électromigration des atomes d'indium eux-mêmes dans une couche d'indium pur. La technique est basée sur la réalisation de zones bien délimitées d'indium radioactif par une radioactivation sélective. Le déplacement après le passage du courant est mesuré, d'une part, par autoradiographie et, d'autre part, par balayage d'un compteur. Les expériences ont été conduites à cinq températures différentes dans le domaine $100-150{ }^{\circ} \mathrm{C}$.

\section{Méthodes expérimentales.}

2.1 GÉOMÉTRIE DES COUCHES MINCES. - Les couches minces d'indium ont la forme en $U$ montrée sur la figure 1 où sont indiquées les dimensions. Leur épaisseur est de $0,3 \mu$. Les zones hachurées 1,2 et 3 sont celles où se trouve initialement l'indium radioactif. Le courant électrique est appliqué entre les deux extrémités. Les atomes subissent le déplacement moyen $l$. Si le phénomène est uniforme le long de tout le ruban, la distance $l_{1-3}$ séparant les taches 1 et 3 


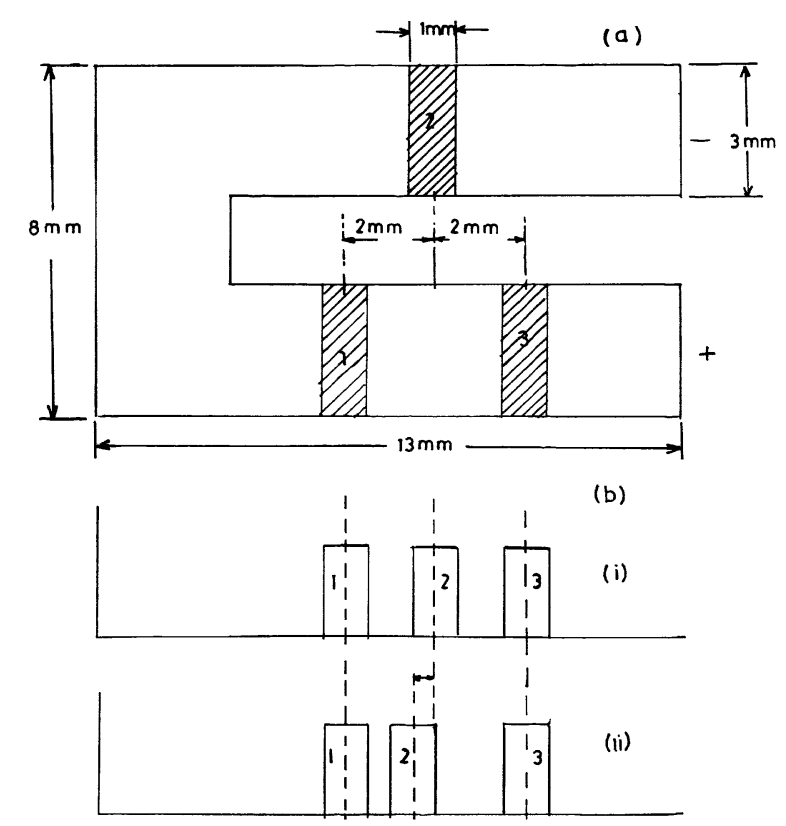

Fig. 1. - Géométrie des rubans pour les mesures d'électromigration. (a) Les zones hachurées sont marquées par des isotopes radioactifs produits par activation neutronique; (b)-(i) Profil théorique de distribution des atomes radioactifs avant le passage du courant ; (ii) profil théorique après le passage du courant en négligeant l'étalement par diffusion.

[(a) Device for the study of electromigration in thin films. The shaded regions contain radioactive material. (b) Radioactive concentration profiles in ideal case without diffusion spread. (i) Before electromigration; (ii) after electromigration.]

doit demeurer constante. Il est facile de constater que la distance $l$ est égale à la moitié de la diminution de la distance $l_{1-2}$ ou la moitié de l'allongement de la distance $l_{2-3}$.

2.2 Radioactivation. - Le substrat est une plaquette de silicium épaisse de $250 \mu$ et recouverte d'une couche d'oxyde d'épaisseur inférieure à $1 \mu$ afin d'assurer une bonne isolation de la métallisation. Une première métallisation d'indium pur à $99,99 \%$ est effectuée à l'aide d'un masque pour recouvrir exactement les trois zones hachurées de la figure 1 , de dimensions égales à $1 \mathrm{~mm} \times 3 \mathrm{~mm}$ et d'épaisseur égale à $0,3 \mu$. Après l'évaporation sous vide, les échantillons sont enveloppés dans une feuille d'aluminium, scellés dans un emballage de polyéthylène, et irradiés dans le réacteur nucléaire C.I.R. du centre atomique de Bombay (Inde) pendant une semaine dans un flux de neutrons thermiques de $10^{14}$ neutrons. $\mathrm{cm}^{-2} \cdot \mathrm{s}^{-1}$. Une partie des atomes d'indium est transformée en In-114 $\mathrm{m}$ de période égale à 50 jours. L'irradiation du substrat de silicium ne donne pas de réaction nucléaire gênante pour la suite.

Une seconde métallisation est alors réalisée à l'aide d'un nouveau masque. Cette fois, un ruban d'indium uniforme et continu, ayant la forme en U (Fig. 1) et une épaisseur d'un micron, est déposé sur le substrat et recouvre les zones 1,2 et 3 .

2. 3 Electromigration. - On réalise des contacts à l'argent aux deux extrémités entre lesquelles un courant continu constant est maintenu à l'aide d'un galvanostat et ajusté de sorte que la densité de courant soit égale à $1,45 \times 10^{4} \mathrm{~A} . \mathrm{cm}^{-2}$ dans toutes les expériences.

La température est régulée et enregistrée pendant toute la durée de l'électromigration, variable entre 1,5 et $78 \mathrm{~h}$. La température est donnée par des thermocouples en contact avec la couche mince. L'ensemble est isolé des éventuelles contaminations extérieures par un tube de silice empli d'argon sous faible pression.

2.4 AUtORADIOGRAPHIES. - L'indium naturel donne par radioactivation neutronique l'isotope $\mathrm{In}-114 \mathrm{~m}$, de période égale à 50 jours et émetteur bêta, donc propice à l'étude autoradiographique. Les rayonnements émis par In-114 m sont très nombreux mais ce sont essentiellement les rayons $\beta^{-}$de $1,98 \mathrm{MeV}$ qui sont utilisés pour l'autoradiographie. Les rayons $\gamma$ $(0,192 \mathrm{MeV}, 0,72 \mathrm{meV}, 0,56 \mathrm{MeV}$ et $0,511 \mathrm{MeV})$ sont peu absorbés par le film photographique. Le pouvoir de résolution de l'autoradiographie est de quelques microns pour les films radiographiques utilisés (films du commerce pour rayons X). Dans les présentes expériences, où le déplacement à mesurer est de l'ordre de $100 \mu$, la précision de la mesure du déplacement (à l'exclusion des autres erreurs sur la durée, la température, le courant, etc.) est donc de quelques $\%$. Un film est appliqué sur le dispositif de la figure 1 avant et après chaque passage du courant électrique. Un exemple est représenté sur la figure 2 avant et après électromigration pendant $50 \mathrm{~h}$ à $134^{\circ} \mathrm{C}$.

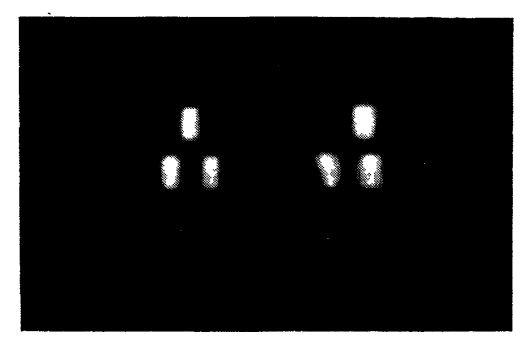

a)

b)

Fig. 2. - Autoradiographie avant et après électromigration de $50 \mathrm{~h}$ à $134^{\circ} \mathrm{C}$. Le déplacement des marques de la figure 1 est bien visible et mesurable.

[Autoradiograph of the film. (a) Not subjected to electrical stress. (b) Subjected to electrical stress for $50 \mathrm{~h}$ at $134{ }^{\circ} \mathrm{C}$.]

Un avantage décisif de l'autoradiographie est de montrer directement sans ambiguité le sens du déplacement : cette autoradiographie montre que les atomes se déplacent comme les électrons vers le pôle positif. On constate en outre clairement que le déplacement 
est uniforme sur tout le ruban : la distance $l_{1-3}$ est invariante. Enfin, on remarque la grande précision de la mesure du déplacement $l$ (égal à la moitié des variations des longueurs $l_{1-2}$ et $l_{2-3}$ ). Dans les conditions de l'expérience, $l$ est compris entre quelques dixièmes et quelques millimètres, mesuré avec un pied à coulisse, en sorte que l'erreur relative sur $l$ est de $1 \%$, ce qui est nettement mieux que la précision des autres méthodes.

2.5 COMPTAGE DE la RAdioaCtivité. - Pour obtenir encore plus précisément les profils de distribution, la radioactivité de l'échantillon est mesurée à travers une fente large de $50 \mu$ et perpendiculaire à la direction du courant. La fente et le compteur (Fig. 3) sont fixes. tandis que l'échantillon est déplacé par pas de $50 \mu$ à l'aide d'une vis micrométrique. La radioactivité de la

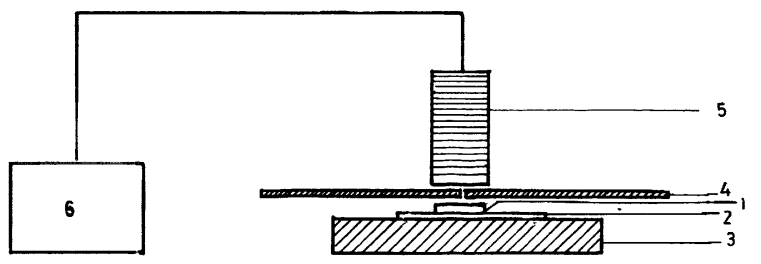

Fig. 3. - Compteur à balayage permettant la mesure du profil de distribution du radiotraceur. 1 : échantillon; 2 : porte-échantillon; 3 : plateforme sur glissière entraînée par une vis micrométrique; $4:$ fente-diaphragme de plomb; 5 : compteur bêta ; 6 : acquisition de données.

[Schematic diagram of tracer scanning arrangement. 1 : sample ; 2 : sample holder 3 : movable platform $; 4$ : adjustable slit; $5: \beta$-detector $; 6:$ analyser.]

portion d'In-114 $\mathrm{m}$ en regard de la fente est ainsi mesurée à l'aide d'un compteur bêta à scintillation plastique. Les profils de concentration enregistrés avant et après le passage du courant permettent de mesurer le déplacement $l$ avec une précision de $1 \%$. Un exemple est représenté sur la figure 4 pour une électromigration de $33 \mathrm{~h}$ à $126^{\circ} \mathrm{C}$. On remarque de nouveau que la distance entre les zones 1 et 3 s'est conservée et que toutes les zones ont subi le même déplacement. La comparaison des profils de chaque zone avant et après électromigration révèle les deux points suivants :

- baisse générale de la radioactivité due à la décroissance de l'isotope In-114 m ( $T=50$ jours);

- élargissement dû à la diffusion. Il faut noter que nous sommes en présence d'une véritable expérience d'autodiffusion.

2.6 OBSERVATIONS AU MICROSCOPE ÉLECTRONIQUE À BALAYAGE. - Le M.E.B. ne permet évidemment pas de mesurer le déplacement des atomes de la matrice. Par contre, il permet d'examiner l'accumulation de matière à l'anode (pôle positif) et la perte de matière à la cathode qui résultent de la conservation de la matière et du déplacement d'ensemble de la

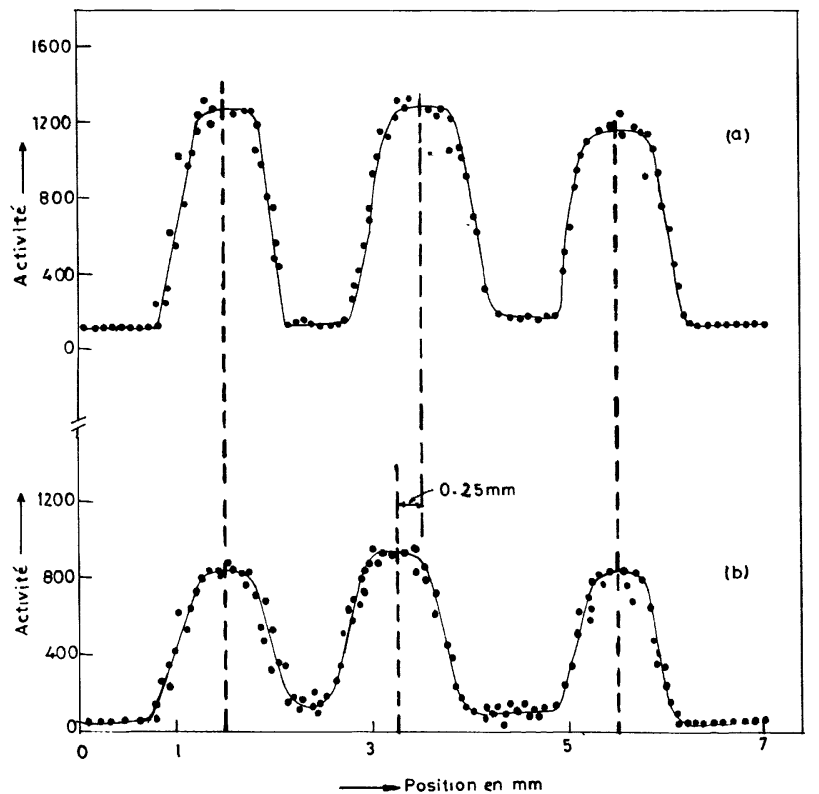

Fig. 4. - Profil de distribution de l'indium radioactif. (a) Avant électromigration. (b) Après électromigration pendant $35 \mathrm{~h}$ à $126^{\circ} \mathrm{C}$.

[Radioactive indium concentration profiles. (a) Before electromigration. (b) After electromigration anneal for $35 \mathrm{~h}$ at $126^{\circ} \mathrm{C}$.]

couche mince dans le sens des électrons. C'est ce que nous pouvons observer sur la figure 5 pour une électromigration de $50 \mathrm{~h}$ à $134^{\circ} \mathrm{C}$. Rappelons que la densité de courant est fixée à $1,4 \times 10^{4} \mathrm{~A} . \mathrm{cm}^{-2}$. Les résultats confirment ceux observés par Kennedy [13] et par Breitling et Hummel [7].

\section{Résultats.}

Nous avons appliqué la méthode précédemment décrite à l'électromigration de films d'indium. Il y a deux raisons à ce choix :-premièrement, l'existence de l'isotope In-114 $\mathrm{m}$ dont le rayonnement (bêta) et la période (50 jours) sont très propices à l'autoradiographie et au comptage à travers une fente-diaphragme; deuxièmement, la faible température de fusion de l'indium permet d'observer des fortes mobilités à température peu élevée. Nous avons ainsi mesuré le déplacement des marqueurs d'indium radioactif dans le domaine des températures comprises entre 100 et $150^{\circ} \mathrm{C}$. Les résultats sont reportés dans le tableau I avec la durée $t$ de l'expérience (d'où la vitesse $v=l / t$ ) et le champ électrique $E$ (d'où la mobilité $\mu=v / E$ ).

La précision peut être estimée à partir des profils de la figure 4. Le déplacement relatif de la zone centrale est mesurée à partir du déplacement du barycentre. Il est égal à $250 \mu \mathrm{m}$ (soit $l=125 \mu \mathrm{m}$ ) avec une incertitude de $2 \mu$. On obtient ainsi $l$ à $1 \%$ près, soit une bien meilleure précision qu'à l'aide du microscope électronique. 


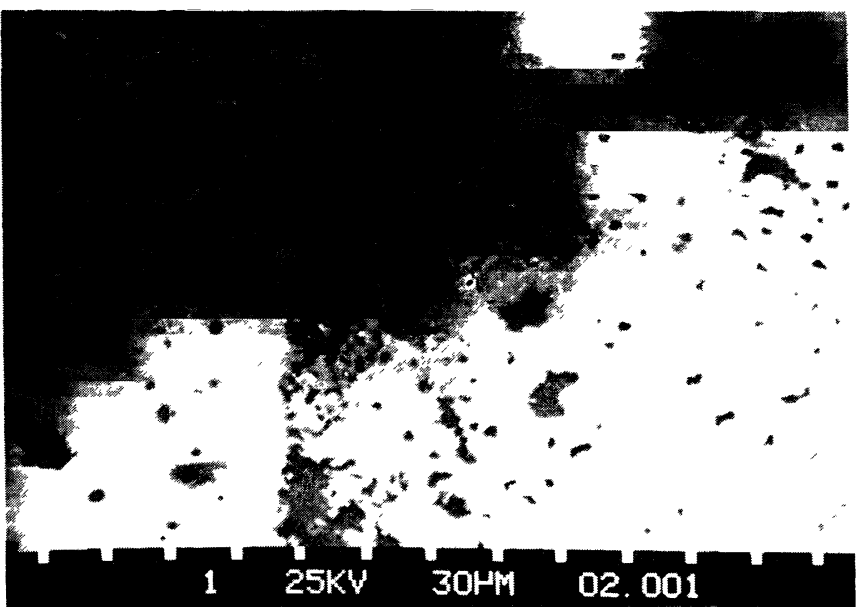

a)

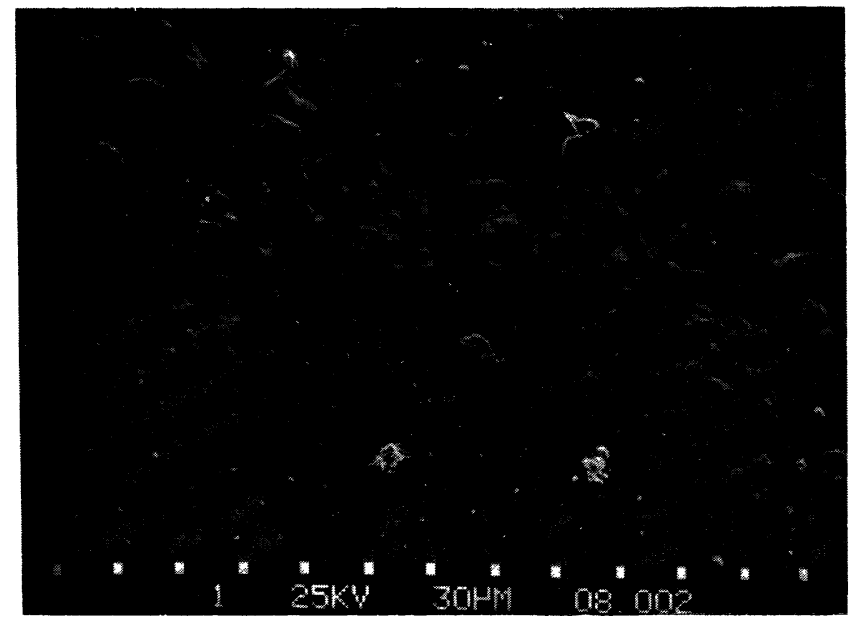

b)

Fig. 5. - Examens au microscope électronique des extrémités du ruban d'indium $(\times 300)$. (a) Accumulation de matière à l'anode. (b) Départ de matière à la cathode.

[Scanning electron microscope pictures of thin indium film after electromigration $\left(40 \mathrm{~h}\right.$ at $\left.134^{\circ} \mathrm{C}\right)$.]

Tableau I. - Mobilité de l'indium déduite des expériences d'électromigration. (La densité de courant est fixée à $1,45 \times 10^{4} \mathrm{~A} \cdot \mathrm{cm}^{-2}$ ).

\begin{tabular}{|c|c|c|c|}
\hline $\begin{array}{c}\text { Tempé- } \\
\text { rature } \\
(\mathrm{K})\end{array}$ & $\begin{array}{c}\text { Durée } \\
(\mathrm{h})\end{array}$ & $\begin{array}{c}\text { Champ } \\
\text { électrique } \\
\left(\mathrm{V} . \mathrm{cm}^{-1}\right)\end{array}$ & $\begin{array}{c}\text { Mobilité } \\
\left(\mathrm{V}^{-1} \cdot \mathrm{cm}^{2} \cdot \mathrm{s}^{-1}\right)\end{array}$ \\
\hline 375 & 78 & 0,36 & $3,69 \times 10^{-8}$ \\
384 & 90 & 0,28 & $5,55 \times 10^{-8}$ \\
399 & 33 & 0,23 & $4,56 \times 10^{-7}$ \\
407 & 40 & 0,24 & $1,23 \times 10^{-6}$ \\
418 & 1,5 & 0,20 & $6,90 \times 10^{-6}$ \\
\hline
\end{tabular}

Les résultats sont représentés graphiquement (Fig. 6) sous la forme d'Arrhénius $\log (\mu T)=f(1 / T)$. On obtient sensiblement une droite d'équation :

$\mu T=3,69 \times 10^{21} \exp (-2,00 / k T) \mathrm{V}^{-1} \cdot \mathrm{cm}^{-2} \cdot \mathrm{s}^{-1} \cdot \mathrm{K}$.

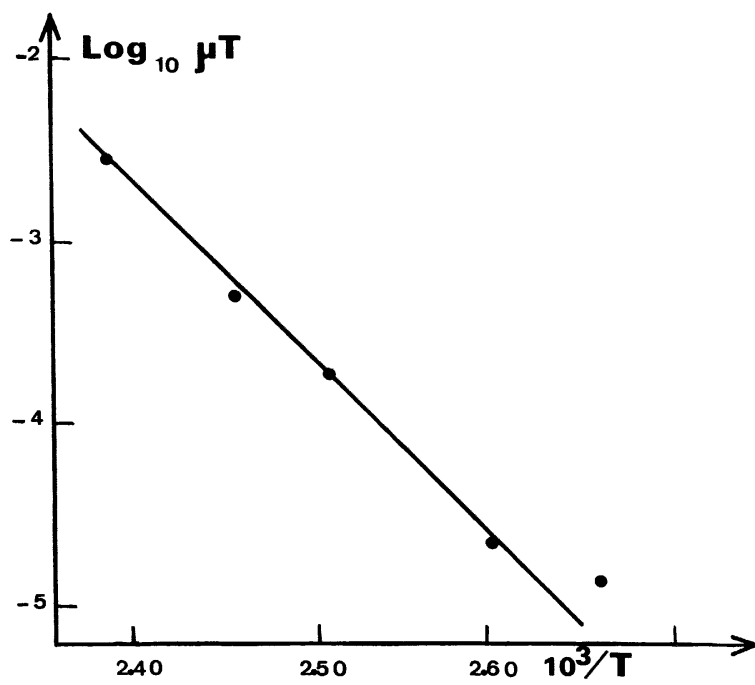

Fig. 6. - Influence de la température sur le produit $\mu T$ (mobilité $\times$ température absolue) pour l'indium en couche mince.

[Temperature dependence on the quantity $\mu T$ (mobility $\times$ absolute temperature), for indium thin films.]

\section{Discussion.}

4.1 Avantages ET INCONVÉNIENTS DE LA MÉTHODE EXPÉRIMENTÉE. - La présente mesure du déplacement d'électromigration possède les qualités suivantes :

- visualisation claire du phénomène grâce au caractère si démonstratif de l'autoradiographie;

- donne sans ambiguité le sens du déplacement (donc le signe de la valence efficace $Z^{*}$ );

- précision de $1 \%$ dans la mesure du déplacement ce qui est largement suffisant compte tenu des autres sources d'erreur : épaisseur de la couche mince, champ électrique, détermination de la température, effet Joule, etc., qui entrainent des incertitudes plus grandes sur la mesure finale de la mobilité.

4.2 Résultats SUR La MOBILITÉ De L'INDIUM. La première constatation que l'on tire de l'examen des autoradiographies et des profils est l'observation de zones parfaitement symétriques après le passage du courant. Cette question est posée depuis que Digiacomo et al. [15] avaient déposé une zone de cuivre sur un ruban d'argent et déterminé le profil de concentration du cuivre à l'aide du MEB. Ils obtenaient des profils asymétriques, ce qui a été interprété par la participation des joints de grain à la diffusion [16]. $\mathrm{Au}$ contraire, les présents résultats et ceux d'autres auteurs $[6,10,12]$ font état de profils symétriques.

Des profils symétriques sont également obtenus sans passage de courant. En effet, le même dispositif expérimental (radioactivation d'une partie du ruban d'indium et analyse de la répartition des atomes radioactifs à l'aide du compteur et d'une fente de $50 \mu)$ a permis d'étudier l'autodiffusion simple sans champ électrique [17]. On observe un profil très 
symétrique, même avec des fortes distances de déplacement $\sim \sqrt{D t} \sim 1 \mathrm{~mm}$.

Le coefficient d'autodiffusion de l'indium sur les mêmes échantillons en couche mince a été mesuré dans le même intervalle de température. Il obéit à la loi d'Arrhénius :

$$
D=0,22 \exp (-0,60 / k T) \mathrm{cm}^{2} \cdot \mathrm{s}^{-1} .
$$

La comparaison avec le coefficient d'autodiffusion de l'indium dans des monocristaux et dans des polycristaux massifs d'indium montre que la diffusion est environ dix fois plus rapide dans les échantillons en couche mince. L'énergie d'activation pour la diffusion $(0,60 \mathrm{eV})$ est inférieure à celle observée dans les échantillons massifs $(0,8 \mathrm{eV})$ mais en accord avec celle obtenue dans des couches minces. Ces résultats de diffusion suggèrent que le transport s'effectue aux joints de grain ou sur les surfaces, en dépit de la forte énergie d'activation $(2 \mathrm{eV})$ observée pour la migration sous champ. Les deux types de mesure doivent être comparés par l'intermédiaire de la relation de NernstEinstein :

$$
\frac{\mu}{D}=\frac{Z^{*} e}{k T}
$$

où $Z^{*}$ désigne la valence efficace, $e$ la charge électronique, $k$ la constante de Boltzmann et $T$ la température absolue. L'analyse détaillée de ces résultats, qui sera prochainement publiée [17], montre que $D$ et $\mu$ ne sont pas idéalement couplés par la loi de NernstEinstein dans le cas des couches minces. La variation apparente de $Z^{*}$ avec la température ainsi que les valeurs très élevées de $Z^{*}$ observées dans de nombreuses autres couches métalliques suggèrent qu'à la limite les atomes puissent se déplacer sous l'action d'une force extérieure sans avoir recours à l'agitation thermique par les défauts de surface. On pourrait imaginer la situation extrême où, à la température de $0 \mathrm{~K}$ et en présence d'un très fort champ électrique, le paramètre $Z^{*}$ résultant de la comparaison du rapport $\mu / D$ tendrait vers l'infini.

\section{Conclusion.}

Nous avons décrit deux méthodes utilisant des isotopes radioactifs pour étudier l'électromigration des atomes constitutifs de couches métalliques minces. Ce phénomène est d'un grand intérêt dans l'industrie en microélectronique en raison de la dégradation des contacts sous l'influence du champ électrique.

Dans ce cas, il s'agit bien d'étudier le transport des atomes de la matrice et, tout comme dans l'autodiffusion, les traceurs radioactifs jouent un rôle essentiel. Nous montrons que les deux méthodes permettent de mesurer avec précision le déplacement par électromigration dans des couches d'indium. Ce métal a été choisi comme exemple en raison de l'existence de son isotope radioactif $\mathrm{In}-114 \mathrm{~m}$ produit par activation neutronique de l'indium naturel.

De nombreux autres métaux pourront être étudiés de la même manière, à condition qu'ils possèdent un isotope radioactif aux propriêtés suivantes :

- production de l'isotope par activation du métal naturel;

- période de décroissance radioactive égale ou supérieure à 1 jour.

La méthode à choisir dépend de la nature du rayonnement :

- autoradiographie pour rayonnement bêta;

- comptage à travers une fente pour rayonnement gamma.

\section{Bibliographie}

[1] Blech, I. A., J.'Appl. Phys. 47 (1976) 1203.

[2] Attardo, M. J. and Rosenberg, R., J. Appl. Phys. 41 (1970) 2381.

[3] Hummel, R. E., Dehoff, R. T. and Geier, H. J., J. Phys. Chem. Solids 37 (1976) 73.

[4] Blech, I. A. and Kinsborn, E., Thin Solid Films 25 (1975) 327.

[5] KLeIN, B. J., J. Phys. F 3 (1973) 691.

[6] Sun, P. H. and Ohring, M., J. Appl. Phys. 47 (1976) 478.

[7] Breitling, H. M. and Hummel, R. E., J. Phys. Chem. Solids 33 (1972) 845.

[8] WeIss, J., Thin Solid Films 13 (1972) 169.

[9] D'Heurle, F. M., Ainslie, N. G., Gangulee, A. and
Shine, M. C., J. Vac. Sci. Technol. 9 (1972) 289.

[10] Beniere, F., Reddy, K. V., Kostopoulos, D. and Le Traon, J. Y., J. Appl. Phys. 49 (1978) 2743.

[11] TAI, K. L., Sun, P. H. and OHRING, M., Thin Solid Films 25 (1975) 343.

[12] Reddy, K. V., Beniere, F., Kostopoulos, D. and Le Traon, J. Y., J. Appl. Phys. 50 (1979) 2782.

[13] Kennedy, D. I., J. Appl. Phys. 39 (1968) 6102.

[14] Hummel, R. E. and Breitling, H. M., Appl. Phys. Lett. 18 (1971) 373.

[15] Digiacomo, G., Peressini, P. and Rutledge, R., J. Appl. Phys. 45 (1974) 1626.

[16] Martin, G., Phys. Status Solidi A 14 (1972) 183.

[17] Prasad, J. J. B., Ph. D. thesis, Madras (Inde) (1983). 